\title{
Size-Dependent Kinetics of Hydriding and Dehydriding of Pd Nanoparticles
}

\author{
Christoph Langhammer, ${ }^{1}$ Vladimir P. Zhdanov, ${ }^{1,2}$ Igor Zorić, ${ }^{1}$ and Bengt Kasemo ${ }^{1}$ \\ ${ }^{1}$ Department of Applied Physics, Chalmers University of Technology, S-412 96 Göteborg, Sweden \\ ${ }^{2}$ Boreskov Institute of Catalysis, Russian Academy of Sciences, Novosibirsk 630090, Russia
}

(Received 24 March 2009; published 31 March 2010)

\begin{abstract}
Using a new indirect nanoplasmonic sensing method with subsecond resolution, we have studied hydriding and dehydriding kinetics of Pd nanoparticles in the size range 1.8-5.4 nm. Strong particle-size effects are observed. The scaling of the hydriding and dehydriding time scales satisfies power and powerexponential laws. The former (with an exponent of 2.9) is in perfect agreement with Monte Carlo simulations of diffusion-controlled hydriding kinetics. The latter is explained by the effect of surface tension on hydrogen desorption from the surface layer. The approach is generalizable to other reactantnanoparticle systems.
\end{abstract}

PACS numbers: 61.46.Df, 66.30.Pa, 68.18.Jk

Hydride formation in metals in general and in $\mathrm{Pd}$, in particular, represents the best example of first-order phase transitions in solids. The conceptual understanding of such phase transitions is based on the Ising-type models. In reality, the corresponding thermodynamics and kinetics are complicated by lattice strain and other factors. During the past decade, these aspects of hydride formation in bulk samples have been extensively studied experimentally and theoretically (for thermodynamics and kinetics, see Refs. [1,2], respectively). Although this subject is still far from exhausted for the bulk, the most recent studies have been oriented towards nanoparticles [3] where the phase separation is observed down to $1.5 \mathrm{~nm}$ [4]. In this limit, new interesting physics is related, e.g., to size effects and surface tension, and there are new potential applications, e.g., for hydrogen storage $[3,5]$.

Concerning applications, we note that hydrogen-based energy systems represent one of the many avenues that are explored for a transition from the present fossil fuel based one. Such systems must solve production and safe storage, distribution and use of hydrogen. The issue at focus here is storage in metal hydrides. Two major challenges in this area are to find suitable stability of hydrides (thermodynamics) and fast enough storage and release kinetics. Both these aspects are addressable by going to nanoparticles. Here, for the first time, we have investigated the kinetics of hydrogen uptake and release for such particles. With our novel indirect nanoplasmonic sensing platform, we have solved the problem of sufficiently fast and sensitive enough kinetics measurements for Pd-particle sizes down to ca. $1 \mathrm{~nm}$, and combined this with modelling.

Beside the metal-hydride physics, chemistry and applications, our results are instructive for, and in several respects generalizable to, the understanding of oxidation, corrosion, surface functionalization, etching, etc., of nanoparticles. In these processes, some molecules (via dissociation on the surface) or atoms in a nanoparticle's environment can reversibly (or irreversibly) enter the bulk of the nanoparticles. For proper combinations of material, (gas- or fluid-phase) molecules and temperature, similar processes as treated here could happen for, e.g., hydrocarbons, ammonia, oxygen, nitric oxides, etc. Technologically important situations where this might play a role are in nanoelectronic components (oxidation, corrosion), heterogeneous catalysis, hydrogen embrittelment of nanostructured materials, and nanoparticle based sensors.

Focusing here on $\mathrm{H}_{2} /$ metal nanosystems, we note that the understanding of the kinetics of hydriding and dehydriding is quite limited, and that available measurements [6] do not provide a basis for scrutinizing the effect of particle size on the kinetics. One reason is that experimental studies are hampered by a lack of reliable, fast and sensitive enough measurement techniques. Typically, the experiments [6] include measurements of pressure change due to hydrogen absorption or release, in Sievert's reactors (a closed system with constant volume) or gravimetric measurements. In addition to problems with temporal resolution, such approaches require large amounts of sample material (0.5-1 g powder) and have to deal with severe deviations from isothermal conditions caused by reaction heat [6]. Our indirect nanoplasmonic sensing platform circumvents these problems, opening up a new avenue for such studies.

Basically (Fig. 1), we measure the optical response of gold nanodisks, which sense dielectric changes in proximal (within $10 \mathrm{~nm}$ ) Pd nanoparticles, as the latter absorb or release hydrogen. The Au nanodisks were deposited on a glass slide by hole-mask colloidal lithography [7] (72 nm diameter, $30 \mathrm{~nm}$ height, $10^{8}$ particles $/ \mathrm{cm}^{2}$ ). The optically active Au nanodisks are separated from the Pd nanoparticles to be studied by a thin dielectric spacer layer. Thus, the Au nanosensors do not themselves physically interact with hydrogen. The actual "sensing", for the case of hydride formation, is achieved through coupling of the strongly enhanced Au-plasmonic near-field to the changing dielectric properties of Pd nanoparticles as they absorb or release hydrogen, similar to plasmonic refractive index 


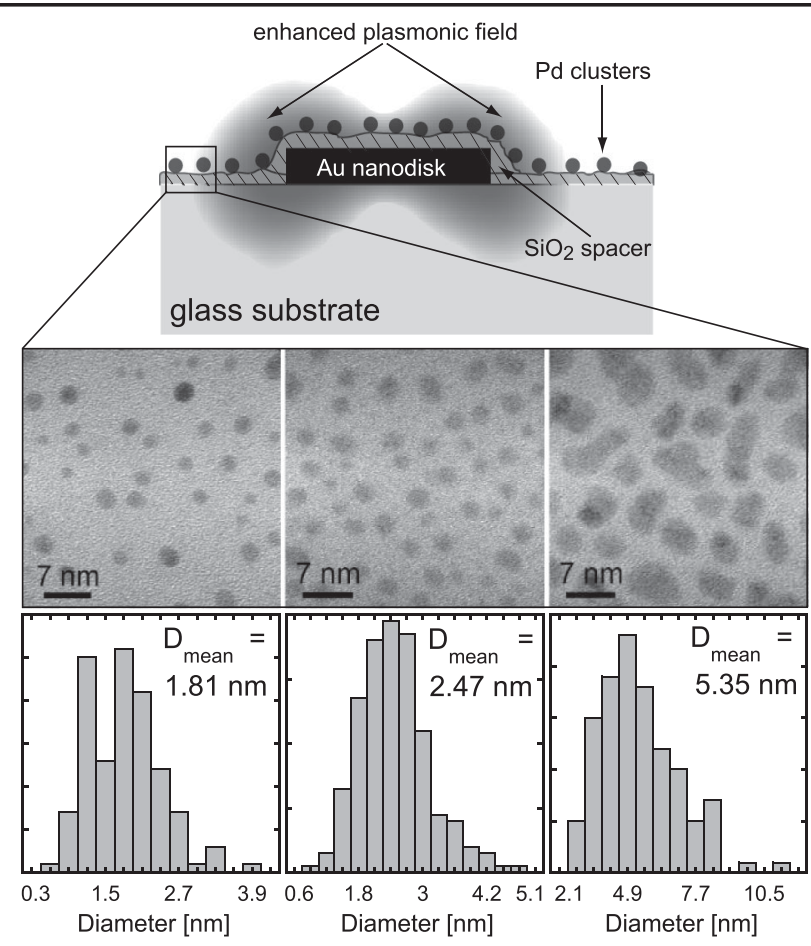

FIG. 1. Scheme of the indirect nanoplasmonic hydrogen sensing platform. The localized surface plasmon resonance (LSPR) of gold nanodisks is used as "a sensor" to characterize a material which undergoes some change upon hydrogen absorption. The latter material (Pd nanoparticles in the present work) is deposited onto a thin dielectric spacer layer, separating the optically active sensor nanodisks from the material to be studied and the hydrogen environment. The change of hydrogen concentration in $\mathrm{Pd}$ nanoparticles is detected trough coupling of the enhanced plasmonic near field of the Au nanodisk plasmons to the changing dielectric properties of $\mathrm{Pd}$ nanoparticles upon $\mathrm{H}$ absorption and desorption. The TEM images below show three examples of Pd nanoparticles, with the corresponding histograms of the size distribution.

based biosening [8]. In particular, the change of the optical extinction, at a particular wavelength $(640 \mathrm{~nm})$, is used as a probe characterizing the Pd nanoparticles upon hydrogen absorption. This signal was shown (by independent QCM measurements) to be proportional to the uptake. Submonolayer coverage of Pd nanoparticles is sufficient for the measurement and thus isothermal conditions prevail (the overheating estimated in analogy with Ref. [9] is negligible). With these specifics, there is excellent control over the whole process, including the particle size, geometry, composition, etc. The remote nature of the sensing makes it applicable under UHV and high pressure conditions. The real-time response enables measurements of subsecond kinetic processes. This is the first time that real-time kinetics of hydriding-dehydriding kinetics have been measured at the nanoscale with such control over the above mentioned factors.

Pd nanoparticles, at submonolayer surface coverage and free from surfactants and stabilizers, were deposited onto the indirect nanoplasmonic sensor surface by electron- beam evaporation of a granular Pd film onto the $\mathrm{SiO}_{2}$ spacer. By varying the thickness of the film, the size of the Pd nanoparticles could be adjusted. After evaporation, the samples were thermally annealed for $3 \mathrm{~h}$ at $220^{\circ} \mathrm{C}$ in Ar, in a furnace and then transferred into a gas tight and temperature-controlled stainless steel cell with optically transparent windows and connected to a vacuum pump. The cell was evacuated and pumped for $12 \mathrm{~h}$ at $105^{\circ} \mathrm{C}$. The nanoparticles were then "activated" by catalytic cleaning, i.e., cyclic exposure to molecular oxygen and hydrogen [18]. Clean nanoparticle surfaces are very important to make sure that the measured kinetics are not governed by surface contamination, but reflect the true response of the formation or decomposition of the hydride phase. After the cleaning step, hydriding-dehydriding cycles were run at $105^{\circ} \mathrm{C}$, until a stable and reversible optical signal was obtained, which was interpreted as the Pd nanoparticles having reached a stable geometrical and structural configuration (i.e., no more hydrogen-induced sintering or restructuring processes, etc.).

Typical TEM images of Pd nanoparticles (with median diameters of $1.81,2.47$, and $5.35 \mathrm{~nm}$ ), resulting from different evaporated film thicknesses, are shown in Fig. 1. The evaporation was performed, at the same time as for the respective real samples, onto $\mathrm{Si}_{3} \mathrm{~N}_{4}$ TEM windows [10], covered with a RF-sputtered $10 \mathrm{~nm} \mathrm{SiO}{ }_{2}$ film, to mimic the spacer layer of the indirect sensing platform. The TEM samples were exposed to the same temperature and hydrogen cycling history, as the real samples.

Because of the presence of a low residual oxygen pressure, it was necessary to work in hydrogen atmosphere all the time, to avoid oxidation of the nanoparticles, as the oxidation also gives rise to a detectable LSPR shift. To measure the hydriding and dehydriding kinetics, the hydrogen pressure in the cell was first adjusted to 1 mbar, where the $\mathrm{H} / \mathrm{Pd}$ ratio is less than 0.005 . Optical extinction was then monitored at a constant wavelength $(640 \mathrm{~nm})$ on the low energy "tail" of the peak of the Au disk LSPR, using a Varian Cary 5000 spectrophotometer with an integration time of $0.05 \mathrm{~s}$. The pressure was then instantly increased to 500 mbar (the cell filling time constant being about $0.06 \mathrm{~s}$ ) and the change in the optical signal was monitored, yielding a hydriding kinetic curve. At $30^{\circ} \mathrm{C}$ and 500 mbar pressure, $\mathrm{Pd}$ is completely converted to its hydride phase, as the plateau pressure for absorption typically is around 20-30 mbar [11] (exceeding the plateau pressure by several hundred mbars corresponds to the absorption regime where the kinetics are insensitive to further pressure increase). After reaching a stable optical extinction signal, attributed to the full conversion of metallic Pd to hydride, the experiment was reversed by instantaneously evacuating the chamber down to 1 mbar hydrogen pressure (evacuation time constant ca $0.25 \mathrm{~s}$ ). The corresponding dehydriding kinetics was again obtained by recording the optical extinction signal in real time. At least 4 hydriding-dehydriding cycles were measured for each nanoparticle size. 
The hydriding (left column) and dehydriding (right column) kinetic curves for Pd nanoparticles with a mean diameter of $1.81,2.47$, and $5.35 \mathrm{~nm}$, measured at $30^{\circ} \mathrm{C}$, are shown in Fig. 2. The normalized variation of the extinction coefficient, proportional to the amount of absorbed hydrogen, is plotted as a function of time. A value of 1 corresponds to $100 \%$ conversion to the hydride phase at 500 mbar hydrogen pressure. The significant difference in signal-to-noise between the curves for different nanoparticle sizes has its origin in decreasing observed LSPR shift for smaller nanoparticles (less material) and thus represents the noise level in the raw data.

The kinetics shown in Fig. 2 exhibit a very strong size dependence and a large difference in the time scales for hydriding and dehydriding. Both features can be explained by different rate limiting steps for hydriding and dehydriding kinetics. The (exothermic) hydriding of a nanoparticle is limited by diffusion of hydrogen atoms from the surface layer to the metallic core via the hydride shell formed in

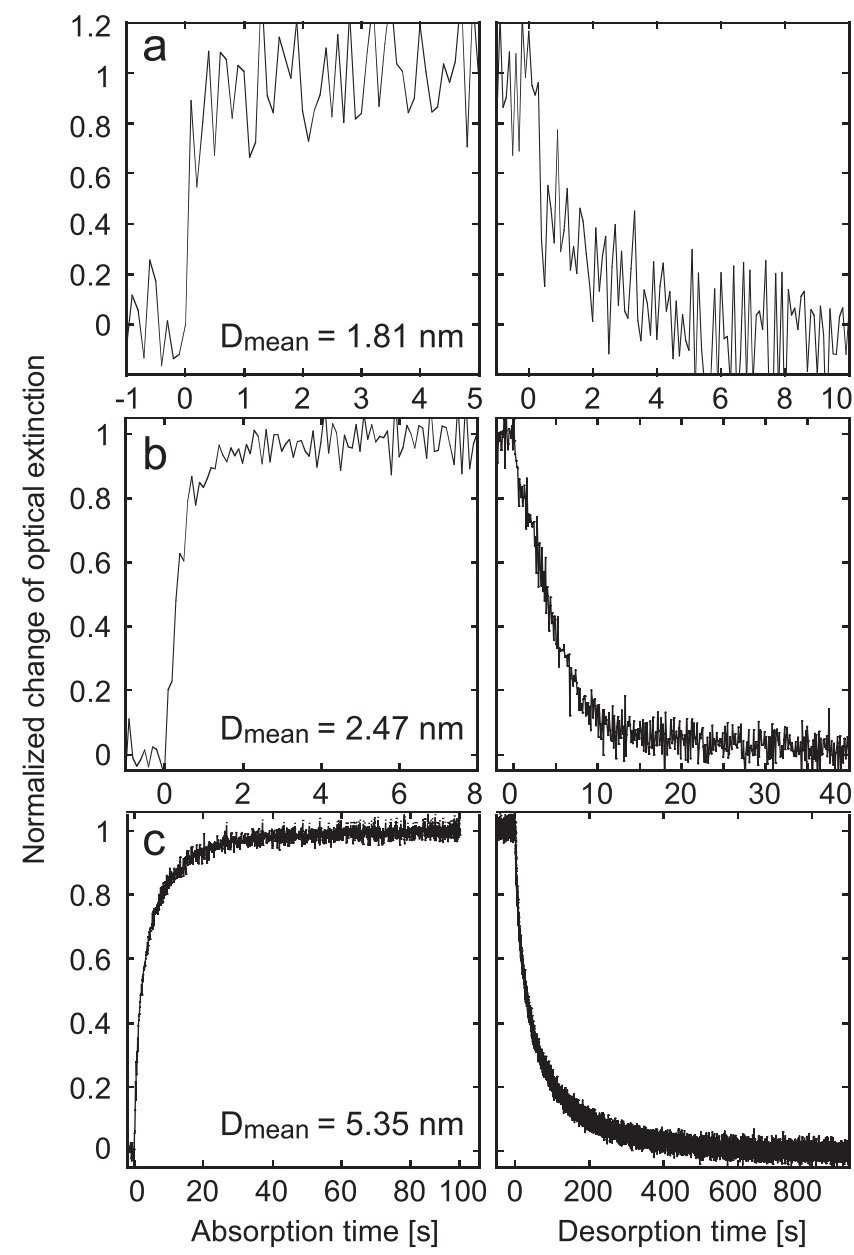

FIG. 2. Experimental hydriding (left column) and dehydriding (right column) kinetics for three mean diameters: $D=1.81$ (a), 2.47 (b), and $5.35 \mathrm{~nm}$ (c) of Pd nanoparticles. The change of the optical extinction, measured at $640 \mathrm{~nm}$, is normalized in such a way that " 1 " corresponds to $100 \%$ conversion of Pd nanoparticles to the hydride phase. this process. The hydride formation itself results from attractive hydrogen-hydrogen interactions. These interactions, influencing diffusion, play a key role in the hydriding kinetics (the role of other factors, e.g., lattice strain is less important here). Monte Carlo simulations [12], taking this effect into account, indicate that the kinetics time scale, $t_{0.5}$ [0.5 corresponds to half of the maximum (saturation) uptake], is proportional to $L^{z}$, where $L$ is the lattice size, and $2.3 \leq z \leq 3$ is the exponent dependent on the diffusion dynamics (the simulations were performed for a cubic lattice; for other lattices, the scale of $z$ is expected to be the same). With a size distribution of nanoparticles, this scaling holds provided that $L$ is replaced by $\langle L\rangle$ [13]. Following this line, we present [Fig. 3(a)] $\ln \left(t_{0.5}\right)$ as a function of $\ln (D)$ ( $D$ is the mean Pd-particle diameter), confirm the power-law scaling, and obtain $z=2.9$ in excellent agreement with simulations [12] performed with physically reasonable diffusion dynamics.

During (endothermic) dehydriding, the hydrogen pressure is low, and hydrogen adsorption is negligible. Initially, a nanoparticle is fully hydrided. The hydride decomposition starts near the surface, the newly formed metallic shell grows, and the hydride core shrinks. This occurs via reversible detachment of hydrogen atoms from the hydride core, their diffusion in the metallic shell, reversible jumps to the surface chemisorption layer, and irreversible associative desorption. For this scenario, the decrease of the number of hydrogen atoms absorbed by a nanoparticle of radius $R$ is described as

$$
d N / d t=-\left(4 \pi R^{2} / \sigma\right) \nu \exp \left(-E_{d} / k_{B} T\right) \theta^{2},
$$

where $4 \pi R^{2} / \sigma$ is the number of adsorption sites ( $\sigma$ is the site area), $\nu$ and $E_{d}$ are the Arrhenius parameters for desorption, and $\theta$ is the hydrogen surface coverage. Since hydrogen diffusion in the metallic shell is rapid (activation energy of only a few $\mathrm{kcal} / \mathrm{mol}$ ) and since the binding energy of adsorbed hydrogen is appreciably larger than in the bulk, we can assume that the hydrogen in the
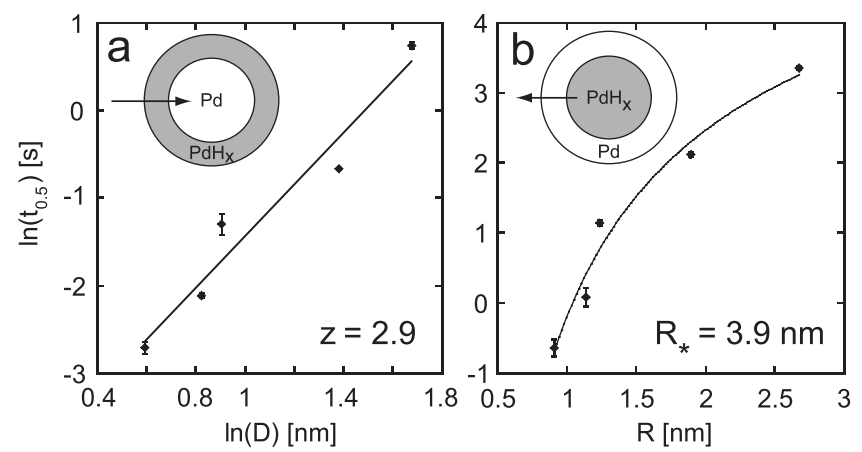

FIG. 3. (a) Power-law scaling of $t_{0.5}$ and $D$ for hydriding. The linear fit corresponds to $z=2.9$. (b) $\ln \left(t_{0.5}\right)$ as a function of $R$ for dehydriding. The solid line represents the best fit according to Eq. (4), yielding $R_{*}=3.9 \mathrm{~nm}$. The error bars are calculated based on 4 independent hydriding and dehydriding kinetic measurements on each sample. 
adsorbed layer is in equilibrium with that in the hydride core, and accordingly, $\theta$ is constant and close to the saturation coverage. In addition, we note that $E_{d}$ depends on the lattice strains related to the presence of hydride and surface tension. The former strain influences only the shape of the kinetic curves [14] while the latter strain influences the dependence of $t_{0.5}$ on $R$. Here, we are interested in the latter dependence and accordingly ignore the former. In this approximation, $E_{d}$ is independent of $N$, and Eq. (1) is integrated as

$$
N=N_{0}-\left(4 \pi R^{2} / \sigma\right) \nu \exp \left(-E_{d} / k_{B} T\right) \theta^{2} t,
$$

where $N_{0} \equiv 4 \pi R^{3} /(3 V)$ is the initial uptake ( $V$ is the volume per hydrogen atom in hydride). $t_{0.5}$ corresponds to $N=N_{0} / 2$, i.e.,

$$
t_{0.5}=\sigma R \exp \left(E_{d} / k_{B} T\right) /\left(6 \nu V \theta^{2}\right) .
$$

In the bulk, the change of the energy of two hydrogen atoms due to surface tension, $\gamma$, is well known to be $\Delta E=$ $4 \gamma v / R$, where $v$ is the partial molar volume of hydrogen in the hydride phase ( $2.6 \AA^{3}$ for Pd). For adsorbed hydrogen, this expression is applicable as well (see, e.g., the DFT calculations [15] illustrating the effect of surface strain on the chemisorption energy). The only difference is that the effective value of $v$ is about twice lower, i.e., $v \simeq$ $1-1.5 \AA^{3}$. Taking into account that $\Delta E$ represents a decrement of the desorption activation energy, we rewrite expression (3) as $t_{0.5}=C R \exp \left(-R_{*} / R\right)$ or

$$
\ln \left(t_{0.5}\right)=\ln (C R)-R_{*} / R,
$$

where $R_{*}=4 \gamma v /\left(k_{B} T\right)$, and $C$ is a constant (with the particle-size distribution, these scaling relations remain valid [13]). Using for Pd $\gamma \simeq 200 \mathrm{meV} / \AA^{2}$ [16] and $v \simeq$ $1-1.5 \AA^{3}$, we obtain $R_{*} \simeq 3-4.5 \mathrm{~nm}$. Figure 3(b) shows that expression (4) with $R_{*}=3.9 \mathrm{~nm}$ is in good agreement with our experimental results.

The kinetics presented were measured at $30{ }^{\circ} \mathrm{C}$. The effect of temperature on the kinetics has been experimentally studied as well (not shown). The apparent activation energies for hydriding and dehydriding $(\simeq 25$ and $65 \mathrm{~kJ} / \mathrm{mol}$ ) are found to be close to those of hydrogen diffusion in hydride and desorption (see, e.g., [17]), respectively. This is in line with the described hydriding and dehydriding scenarios.

In summary, using a new indirect nanoplasmonic sensing platform, we have studied the size dependence of the hydriding and dehydriding kinetics in Pd nanoparticles (for the corresponding absorption isotherms, see Ref. [19]). The results obtained are in excellent agreement with Monte Carlo simulations of hydriding kinetics [12] and our here presented mean-field model of dehydriding ki- netics. In the latter case, the effect of surface tension is demonstrated to be especially important to understand how the time scale of the kinetics depends on nanoparticle size. Experimentally and theoretically, our approach is extendable to other situations, where nanoparticles absorb and desorb atoms of a single kind from a surrounding medium. In fact, our platform allows us to study kinetic processes not only in the bulk but also on the surface of nanoparticles (e.g., during kinetic phase transitions in $\mathrm{CO}$ or $\mathrm{H}_{2}$ oxidation [20]).

We acknowledge Eva Olsson and Stefan Gustafsson for the TEM pictures and the Swedish Energy Agency for financial support (project NANO-SEE 181-1).

[1] R. B. Schwarz and A. G. Khachaturyan, Phys. Rev. Lett. 74, 2523 (1995); E. A. Brener, V. I. Marchenko, and R. Spatschek, Phys. Rev. E 75, 041604 (2007).

[2] V. P. Zhdanov, A. Krozer, and B. Kasemo, Phys. Rev. B 47, 11044 (1993); A. Borgschulte, R. Gremaud, and R. Griessen, Phys. Rev. B 78, 094106 (2008).

[3] V. Berube et al., Int. J. Energy Res. 31, 637 (2007); M. Yamauchi et al., Chem. Phys. Chem. 10, 2566 (2009).

[4] H. Jobic et al., J. Less-Common Met. 129, 311 (1987); B. Ingham et al., Phys. Rev. B 78, 245408 (2008); D. G. Narehood et al., Int. J. Hydrogen Energy 34, 952 (2009).

[5] M. Dornheim et al., Adv. Eng. Mater. 8, 377 (2006).

[6] K. S. Nahm et al., Int. J. Hydrogen Energy 17, 333 (1992); M. Martin et al., J. Alloys Compd. 238, 193 (1996); A. Inomata et al., J. Alloys Compd. 278, 103 (1998); G. Liang et al., J. Alloys Compd. 305, 239 (2000); Q. Li et al., J. Alloys Compd. 373, 122 (2004).

[7] H. Fredriksson et al., Adv. Mater. 19, 4297 (2007).

[8] J. N. Anker et al., Nature Mater. 7, 442 (2008).

[9] V. P. Zhdanov and B. Kasemo, Catal. Lett. 75, 61 (2001).

[10] A. W. Grant et al., Nanotechnology 15, 1175 (2004).

[11] C. Langhammer et al., Nano Lett. 7, 3122 (2007).

[12] V. P. Zhdanov and B. Kasemo, Chem. Phys. Lett. 460, 158 (2008).

[13] V. P. Zhdanov and B. Kasemo, Physica (Amsterdam) 42E, 1482 (2010).

[14] V. P. Zhdanov and B. Kasemo, J. Phys. Chem. C 113, 6894 (2009).

[15] M. Mavrikakis, B. Hammer, and J. K. Norskov, Phys. Rev. Lett. 81, 2819 (1998); I. V. Yudanov et al., J. Phys. Chem. C 112, 20269 (2008).

[16] J. Kollar et al., Phys. Rev. B 68, 245417 (2003); R. Lamber, S. Wetjen, and N. I. Jaeger, Phys. Rev. B 51, 10968 (1995).

[17] G. E. Gdowski et al., Surf. Sci. 181, L147 (1987); M. Morkel et al., Surf. Sci. 588, L209 (2005).

[18] V. Johanek et al., Science 304, 1639 (2004).

[19] C. Langhammer et al., Chem. Phys. Lett. 488, 62 (2010).

[20] E. M. Larsson et al., Science 326, 1091 (2009). 